Applied and

NISTIR 5124

Computational

Mathematics

Division

Computing and Applied Mathematics Laboratory

\title{
Computation of Complex Solidification Morphologies Using a Phase-Field Model
}

\author{
B. T. Murray, A. A. Wheeler, W. J. Boettinger \\ and G. B. McFadden
}

February 1993

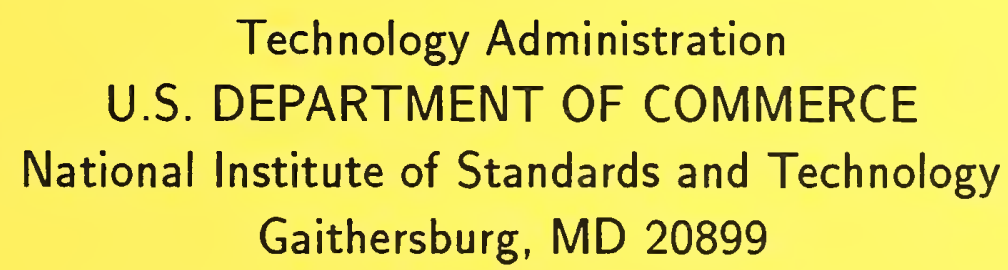

QC

100

.456 



\section{Computation of Complex Solidification Morphologies Using a Phase-Field Model}

\author{
B. T. Murray \\ A. A. Wheeler \\ W. J. Boettinger \\ G. B. McFadden
}

U.S. DEPARTMENT OF COMMERCE

Technology Administration

National Institute of Standards

and Technology

Applied and Computational Mathematics Division Computing and Applied Mathematics Laboratory Gaithersburg, MD 20899

February 1993

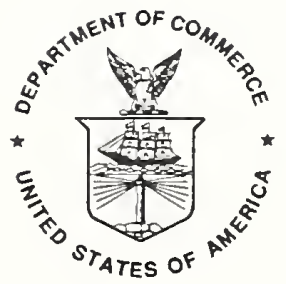

U.S. DEPARTMENT OF COMMERCE Ronald H. Brown, Secretary

NATIONAL INSTITUTE OF STANDARDS

AND TECHNOLOGY

John W. Lyons, Director 



\title{
Computation of Complex Solidification Morphologies Using a Phase-Field Model
}

\author{
B. T. Murray, A. A. Wheeler, W. J. Boettinger, and G. B. McFadden \\ National Institute of Standards and Technology \\ Gaithersburg, MD 20899 USA
}

\begin{abstract}
An anisotropic phase-field model is used to calculate numerically the solidification patterns of a pure material into an undercooled liquid in a two-dimensional rectangular region. In the phase-field approach, the solid-liquid interface is treated as diffuse, and a dynamic equation for the phase variable is introduced in addition to the equation for heat flow. The phase-field model equations are solved using finite-difference techniques on a uniform mesh. Calculations for dendritic growth are presented for both four-fold and six-fold anisotropy, and the effect of the level of anisotropy on the growth of a dendrite is investigated. A previous study has shown that performing computations with an interface that is sufficiently thin for the numerical solution to accurately represent a sharp interface model is computationally demanding. However, even with a relatively thick interface, the computations using the phase-field model show many of the qualitative features of dendritic growth, and the method is well suited for handling the evolution of very complex, realistic in terface shapes.
\end{abstract}

*School of Mathematics, University of Bristol, Bristol BS8 1TW, U.K. 


\section{Introduction}

Phase-field models provide a comparatively new setting for the theoretical investigation of phase transitions, such as solidification of a pure material. The classical free boundary approach models phase change between two phases separated by a moving interface upon which boundary conditions must be prescribed. In contrast, the phase-field method introduces a continuous transition between the two phases across a thin layer of finite thickness. An additional variable called the phase field is introduced whose value at a point identifies the phase. The advantage of this approach lies in its coherent description of phase change, whereby no distinction is made in the model between the solid, liquid or interfacial layer. An important consequence of the phase-field approach is that numerical solutions of the resulting equations can yield complicated growth morphologies whose connectedness changes in time; thus, it is well suited for the numerical simulation of complicated phenomena such as dendritic growth, a situation which is very difficult to solve numerically employing algorithms based on the classical free-boundary problem formulation.

Early work on the development of phase-field models for first-order phase transitions can be traced back to a number of investigators [1-5]. The development of a field equation for an order parameter or phase-field variable that can be used to model solidification borrows ideas from the related physical problems of diffuse phase boundaries and critical point phenomena [6-9]. Several computations using a phase-field model have been performed for one-dimensional planar or radially symmetric geometries [10-13]. Recently, Kobayashi $[14,15]$ reported computations of a phase-field model for a pure material in two and three spatial dimensions, which clearly showed the evolution of solid dendritic structures into an undercooled melt. Kobayashi's work was the first qualitative demonstration of the possible utility of phase-field models of solidification as a computational tool for modeling complicated, realistic solid/liquid interfaces.

More recently, a phase-field model has been developed and used to numerically simulate the solidification of a pure material $[16,17]$. This model was formulated in a manner consistent with irreversible thermodynamics [18] and careful attention was paid to the determination of the phase-field model parameters in terms of the classical material parameters. This recent model is also well suited to numerical computation. There has also been some 
recent work on the development of a phase-field model for the solidification of an alloy in an isothermal system $[19,20]$. The methodology employed in developing these newer models has provided the necessary framework for the development of a complete phase-field description for the more general treatment of a nonisothermal binary alloy.

In this paper, additional computations for a two-dimensional region are performed using the model developed recently for a pure material. The time-dependent heat and phase-field equations are solved numerically using finite-difference techniques on a uniform computational mesh for conditions simulating dendritic growth into an undercooled liquid. Computations are performed for both four-fold and six-fold anisotropy. Some qualitative aspects of using the phase-field approach for such calculations is investigated. This work is part of an ongoing effort to develop and evaluate phase-field models of solidification.

\section{Governing Equations}

Phase-field models of solidification are typically derived from a Landau-Ginzburg or CahnHilliard type of free-energy functional:

$$
\mathcal{F}=\int_{V}\left[f(\phi, T)+\frac{\epsilon^{2}}{2}(\nabla \phi)^{2}\right] d V
$$

where $V$ is the region occupied by the system, $\phi(\mathbf{x}, t)$ is the phase-field, $T(\mathbf{x}, t)$ is the temperature and $\epsilon$ is a gradient energy coefficient, which is constant for an isotropic material. The free-energy density $f(\phi, T)$ is a monotonic function of $T$ and is a double-well with respect to $\phi$, which yields two minima with respect to $\phi$ representing the free energy of the solid and liquid phases for each temperature. Different choices for the precise functional form of $f$ have been used in the various earlier models $[4,15]$. In most of the previous developments of phase-field models, the free energy Eq. (1) has been used to obtain a kinetic equation for the phase field by requiring that it evolves in a manner such that the total free energy $\mathcal{F}$ decreases monotonically in time. The simplest form of a kinetic equation that is consistent with this requirement is the following:

$$
\frac{\partial \phi}{\partial t}=-M_{1} \frac{\delta \mathcal{F}}{\delta \phi}=-M_{1}\left(\frac{\partial f}{\partial \phi}-\epsilon^{2} \nabla^{2} \phi\right)
$$

where $M_{1}$ is a parameter that can be related to the interface kinetic coefficient of the sharp interface problem. Typically in the formulation of a phase-field model, a modified form of 
the heat equation is assumed which includes an appropriate source term to account for the liberation of latent heat, e.g.,

$$
\frac{\partial T}{\partial t}+K \frac{\partial \phi}{\partial t}=\kappa \nabla^{2} T
$$

where $K$ is a constant proportional to the latent heat per unit volume, and $\kappa$ is the thermal diffusivity.

Penrose and Fife [18] have addressed the problem of deriving the set of equations in a more rigorous fashion by employing an an entropy functional, which is the appropriate thermodynamic potential for nonisothermal situations. They derived the set of phase-field equations using a form for the potential, $f(\phi, T)$, introduced in earlier studies [3,4]. However, their choice for the form of the potential function, $f$ has the disadvantage that the values of $\phi$ representing the solid and liquid states depends upon temperature and other model parameters. This limitation complicates the implementation of the phase-field model for practical computation.

A recently developed phase-field model is used for the calculations here which is derived in [17]. The model is derived from an entropy functional in a manner similar to Penrose and Fife [18], but it is constructed so that the states $\phi \equiv 0$ and $\phi \equiv 1$ correspond to the bulk solid and liquid phases, respectively, independent of the temperature. This has the advantage that latent heat is liberated only in the interfacial region. It is noted here that different conventions are used for the values of $\phi$ corresponding to the bulk phases in the various phase-field models. This new phase-field model results in the following dimensionless governing equations for the temperature and phase field:

$$
\begin{gathered}
\frac{\partial u}{\partial t}+\frac{30 g(\phi)}{S} \frac{\partial \phi}{\partial t}=\nabla^{2} u, \\
\frac{\tilde{\epsilon}^{2}}{m} \frac{\partial \phi}{\partial t}=30 g(\phi) \tilde{\epsilon} \alpha S u-\frac{1}{4} g^{\prime}(\phi)+\tilde{\epsilon}^{2} \nabla^{2} \phi .
\end{gathered}
$$

where $g(\phi)=\phi^{2}(1-\phi)^{2}$ and prime denotes differentiation. Here, length has been scaled on some reference length scale $w$ of say the dimensions of the domain, time on the corresponding thermal diffusion time $w^{2} / \kappa$, and temperature by putting $T=T_{M}+\Delta T u$, where $T_{M}$ is the equilibrium melting temperature and $\Delta T$ is a reference temperature difference (such as, between the melting temperature and the initial temperature at the boundary of the domain). 
This isotropic phase-field model is characterized by four dimensionless parameters:

$$
\begin{gathered}
S=\frac{c \Delta T}{L}, \\
\alpha=\frac{\sqrt{2} w L^{2}}{12 c \sigma T_{M}}, \\
m=\frac{\mu \sigma T_{M}}{\kappa L},
\end{gathered}
$$

and

$$
\tilde{\epsilon}=\frac{\delta}{w}
$$

where $c$ is the specific heat per unit volume, $L$ is the latent heat per unit volume, $\sigma$ is the surface energy, $\mu$ is the interface kinetic coefficient, and $\delta$ is a measure of the interface thickness, which has been related to the gradient energy coefficient, $\epsilon$, by analyzing a one-dimensional equilibrium solution of the dimensional phase-field equation. In [17], it is shown how the phase-field parameters $\alpha$ and $m$ are related to the physical parameters which characterize the interface dynamics (i.e., interfacial energy and kinetic coefficient). Once the characteristic length scale $w$ has been chosen, knowledge of the physical properties leaves one degree of freedom, namely $\tilde{\epsilon}$, which then is used to set the interface thickness. It is expected that in order to model the physical behavior correctly, the interface thickness must be sufficiently small compared to the interfacial macrostructures that are to be modeled; however, from a computational viewpoint, it is desirable for the interface thickness to be as large as possible in order that accurate solutions of the phase-field equations can be obtained for practical computational effort.

In order to simulate dendritic growth, the phase-field equation given by Eq. (5) is modified to include anisotropy in the parameter $\tilde{\epsilon}$ by introducing

$$
\tilde{\epsilon}(\theta)=\bar{\epsilon} \eta(\theta)=\bar{\epsilon}(1+\gamma \cos k \theta) .
$$

The angle $\theta$ is defined as the angle between the normal to the interface and the $x$-axis, and $k$ specifies the mode number. Rederiving Eq. (5) after including the variation of the gradient energy coefficient with orientation yields a modified version of the phase-field equation:

$$
\begin{aligned}
\frac{\bar{\epsilon}^{2}}{m} \frac{\partial \phi}{\partial t}=\left\{\phi ( 1 - \phi ) \left[\phi-\frac{1}{2}+30 \bar{\epsilon} \alpha S\right.\right. & \phi(1-\phi) u]-\bar{\epsilon}^{2} \frac{\partial}{\partial x}\left(\eta(\theta) \eta^{\prime}(\theta) \frac{\partial \phi}{\partial y}\right) \\
& \left.+\bar{\epsilon}^{2} \frac{\partial}{\partial y}\left(\eta(\theta) \eta^{\prime}(\theta) \frac{\partial \phi}{\partial x}\right)+\bar{\epsilon}^{2} \nabla \cdot\left(\eta^{2}(\theta) \nabla \phi\right)\right\} .
\end{aligned}
$$


In the phase-field model, the interface is represented by a range of contour levels of $\phi$, e.g., $0.1 \leq \phi \leq 0.9$. In order to compute the anisotropic behavior, the orientation angle is determined in terms of the phase field $\phi$ using the following relation for the normal vector:

$$
\hat{n}=\frac{\nabla \phi}{|\nabla \phi|}=\cos \theta \hat{x}+\sin \theta \hat{y} \text {. }
$$

From this expression we have the definition

$$
\tan \theta=\frac{\phi_{y}}{\phi_{x}}
$$

and, in addition, we obtain

$$
\theta_{x}=\frac{\phi_{x} \phi_{x y}-\phi_{y} \phi_{x x}}{|\nabla \phi|^{2}}, \quad \theta_{y}=\frac{\phi_{x} \phi_{y y}-\phi_{y} \phi_{x y}}{|\nabla \phi|^{2}} .
$$

These relations are used to compute the terms in Eq. (10) which arise from expanding the derivatives of $\eta(\theta)$.

By conducting an asymptotic analysis of the phase-field equations in the limit $\tilde{\epsilon} \rightarrow 0$ with $S, \alpha$, and $m$ of order one, the classical description of an appropriate free boundary problem is recovered. The details are analogous to those given by Caginalp [5] for the form of the free energy investigated by him, and a thorough development of the interfacial conditions for the present phase-field model with the assumed form for $\tilde{\epsilon}(\theta)$ is given in [21]. The free boundary problem satisfied by the leading-order temperature for the phase-field model given by Eq. (4) and Eq. (10) is

$$
\frac{\partial u}{\partial t}=\nabla^{2} u
$$

with interfacial boundary conditions

$$
\left[\frac{\partial u}{\partial n}\right]_{\text {Solid }}^{\text {Liquid }}=-\frac{v_{n}}{S}
$$

and

$$
u=-\Gamma\left\{\frac{v_{n}}{m[\eta(\theta)]^{2}}+\left[\eta(\theta)+\eta^{\prime \prime}(\theta)\right] \mathcal{K}\right\},
$$

where $\Gamma=\sigma T_{m} /[w L \Delta T]$ is the dimensionless capillary length, $v_{n}$ is the dimensionless normal velocity of the interface into the liquid and $\mathcal{K}$ is the dimensionless interfacial curvature (measured positive for convex projections into the liquid). The interfacial boundary condition Eq. (13) reduces to the anisotropic form of the Gibbs-Thomson equation when $v_{n}=0$; the term including $v_{n}$ incorporates the effect of interface kinetics. Note that allowing $\tilde{\epsilon}$ to depend on $\theta$ modifies both the term proportional to velocity and the term proportional to curvature. 


\section{Numerical Computations}

The objective here is to evaluate the behavior of the phase-field model by simulating the growth of a two-dimensional dendrite into an undercooled liquid. In order to perform the simulation, the governing equations are solved numerically in a two-dimensional rectangular domain of sufficient size to allow for the development of characteristic dendritic structure (e.g., side arms). Even with the phase-field approach, an optimum solution procedure should employ some type of adaptive technique, particularly for the phase variable, since it varies over a very small part of the domain. However, we chose at present to use straightforward finite difference solution techniques applied on a uniform computational grid, since the numerical implementation of such techniques is straightforward and they are well suited for taking full advantage of highly vectorized large-scale computers.

The governing equations given by Eq. (4) and Eq. (10) are a pair of coupled, secondorder, nonlinear parabolic equations. They are discretized spatially using second-order finite differences on a uniform grid characterized by mesh spacings $\Delta X$ and $\Delta Y$ in the $x$ and $y$ coordinate directions, respectively; for the temporal discretization we introduce the time step $\Delta t$. In order to maximize the computational efficiency, we employ explicit time-differencing on the $\phi$ equation which is nonlinear in all terms except for the highest-order spatial derivatives. The heat Eq. (4) is linear in the temperature $u$ but contains the source term depending on $\phi$. For the present calculations, the thermal field diffuses more rapidly, so to avoid the diffusive stability requirement, the alternating-direction implicit method (ADI) is used on Eq. (4). The methods employed here are described in many standard texts on finite difference techniques for partial differential equations (e.g., [22]).

For the two-dimensional rectangular computational domain, vanishing Neumann conditions for both $\phi$ and $u$ are applied at the boundaries, which have the scaled dimensions of $X_{L}$ and $Y_{L}$ in the $x$ and $y$ coordinate directions, respectively. We note that some of the results presented here for the temperature and phase field are displayed after reflecting the computational domain about the line $y=0$, which corresponds to the axis of the dendrite. From the chosen definition of the dimensionless variables, the value $u=0$ corresponds to the melting temperature of the pure material, while $u=-1$ is the initial undercooling temperature (the dimensional undercooling is $\Delta T$ ). Initially for each calculation, a small region 
of solid $(\phi=0, u=0)$ is located at the $x=0, y=0$ corner of the domain, and the remainder of the domain is undercooled liquid $(u=-1)$. The shape of the initial solid region is one-quarter of a circle with an initial radius denoted by $r_{0}$.

The two-dimensional simulations were performed using property values for pure nickel; the values for the required material parameters are readily available (see for example [20]). The property values used to determine the dimensionless parameters for the computations are $\sigma=3.7 \times 10^{-5} \mathrm{~J} / \mathrm{cm}^{2}, T_{m}=1728 \mathrm{~K}, L=2350 \mathrm{~J} / \mathrm{cm}^{3}, c=5.42 \mathrm{~J} / \mathrm{K} \mathrm{cm} \mathrm{cm}^{3}, \kappa=0.155 \mathrm{~cm}^{2} / \mathrm{s}$, and $\mu=285 \mathrm{~cm} / \mathrm{K}$ s. In order to completely determine the dimensionless parameters Eq. (7) - Eq. (9), we must choose values for the reference length, $w$, and the interface thickness $\delta$. The choice of these parameters is based on the physical structure we wish to compute and the practical limitations of accurately resolving gradients within the interfacial region for a desired computational domain of size $X_{L}$ and $Y_{L}$.

Because the computational domain is thermally insulated, the value of the dimensionless undercooling $S$ determines the portion of the domain which can be solidified. The computations are facilitated by larger values of $S$ (large values of undercooling, $\Delta T$ ) because the solid encompasses a larger portion of the computational domain. In addition, a larger value of $S$ produces a thinner thermal boundary layer in the liquid so the effect of the adiabatic outer boundaries is less pronounced. A value of $S=0.5$ is used for all the calculations presented. This value corresponds to an actual undercooling of $217 \mathrm{~K}$, which is an attainable level of undercooling for nickel. Based on some preliminary computational experiments to determine how the interface thickness and the size of the domain should be chosen in relation to the tip radius, we chose a length scale $w=2.1 \times 10^{-4} \mathrm{~cm}$ which yields the parameter value $\alpha=400$ in the definition Eq. (7). For the physical parameters of nickel given above, the dimensionless parameter $m$ has the value 0.05 .

With the parameters $S, \alpha$ and $m$ specified, the parameter $\bar{\epsilon}$ determines the thickness of the interface. The computational resolution was determined by the extent of the domain chosen $\left(X_{L}\right.$ and $\left.Y_{L}\right)$ and the number of grid points used in the discretization of the domain. For all the computations presented here, $\tilde{\epsilon}=0.005$ is used; this value was found to give an optimal interface width from the point of view of computational efficiency for the present parameters and computation scheme. A more complete discussion about the choice of the model parameters and the effect of varying the interface thickness is given in [16]. 


\section{Results and Discussion}

In Wheeler et al. [16], a quantitative evaluation of the phase-field model for one- and twodimensional computations was performed by investigating the effects of interface width and spatial and temporal numerical resolution. For one-dimensional spherically symmetric calculations, very good agreement was obtained between the phase-field results and computations from the corresponding sharp interface formulation solved numerically using a Green's function approach. The phase-field model was evaluated by comparison to existing sharp interface theories of two-dimensional dendritic growth. Exact comparisons were not possible owing to the differences in the details included in the theories, but the phase-field calculations were shown to produce behavior which is in agreement with the more well-established theories, i.e., the Ivantsov/microscopic solvability theories.

Here, we present some additional computations which display the complexity of the solidification structures that can be produced by the phase-field model and show that they give a qualitative representation of dendritic growth behavior. Similar computations were performed by Kobayashi [15] using a related phase-field model. The contribution of the present calculations is that they are obtained from a phase-field model which has been analyzed in considerable detail and for which the model parameters have been well-established $[16,17,21]$.

The results presented are displayed as contours of the temperature and phase field. In all cases, for each temperature plot eleven isotherms are shown which are fractions of the maximum magnitude of the temperature $(0.05,0.1-0.9$, and 0.95$)$. In the phase field plots, the interface is represented by contours in the range $0.1 \leq \phi \leq 0.9$ which are indistinguishable.

In figs. 1 and 2, we present computations which illustrate the role played by anisotropy in the parameter $\tilde{\epsilon}$. Both calculations were performed on half the domain shown with $X_{L}=$ 4.5 and $Y_{L}=2.25$ with 600 grid points in the $x$ direction and 300 points in the $y$ direction $(\Delta X=\Delta Y=0.0075)$. For these calculations, a time-step of $5 \times 10^{-5}$ was used. In the figures for the purpose of illustration, the $x$ direction has been oriented so that it is vertical and the solution has been reflected about the center vertical line $(y=0)$. Fig. 1 shows the temperature and phase-field contours at three dimensionless time levels in the computation $(t=0.1,0.3$, and 0.5$)$ for the case when there is no anisotropy $(\gamma=0)$. The initially circular 
solid region is subject to morphological instability and breaks down into a number of fingerlike structures which grow and experience further instability in the form of tip-splitting. With four-fold anisotropy $(k=4)$ and an anisotropy parameter value, $\gamma=0.02$, fig. 2 shows the temperature and phase-field contours at the same three dimensionless time levels as in fig. 1. From the definition of the orientation angle, the preferred growth directions for $k=4$ are along the coordinate axes. Additionally, there is a short solid finger with an orientation of approximately 45 degrees which has grown out early in the calculation. After an initial transient period, the tips of the needle crystals reach a well-defined operating state with a particular radius and growth velocity. These values remain constant until the proximity of the outer boundary of the domain inhibits growth.

In fig. 3 isotherms and phase-field contours at a single time level of 0.6 are plotted for a lower value of the anisotropy parameter, $\gamma=0.01$. Again, because of the influence of anisotropy, a well-defined needle crystal is obtained. However, in this case, regular sidebranching occurs at a distance behind the tip of the growing crystal. The occurrence of side branching is found to depend on the anisotropy level ( $\gamma$ value), and to some extent on the numerical error; the side-branched structure occurs more readily as the discretization error increases. For this set of calculations, the mesh spacing $\Delta X=\Delta Y=0.015$ was used and the time-step was $1 \times 10^{-4}$. The dependence of side-arm formation on anisotropy level was also displayed by the simulations of Saito, et al. [23], which were performed using a boundary integral approach to solve a sharp interface model with a quasistationary heat equation and no kinetic effects.

Kobayashi [15] found that the occurrence of side-branched structure is enhanced by introducing random noise into the calculations. For the subsequent calculations presented, random noise of small amplitude has been introduced into the temperature term in the phase-field equation Eq. (10), which represents a disturbance to the interface. Fig. 4 is a calculation with anisotropy, $\gamma=0.01$, and with a $1 \%$ random noise level. Isotherms and phase-field contours are shown at four time levels which are equally spaced except for the lowest two time levels. At the lowest time level shown, the preferred directions along the coordinate axes experience the fastest growth and the solid no longer resembles the initially circular region. At the second time level, side-arm formation has begun to occur, and in the last two time levels the side-branched dendritic structure is clearly evident. The presence 
of the random noise does not effect the overall growth conditions of the needle crystal (tip radius and velocity), but does enhance the irregular production of the secondary arms. Because the actual computational domain is shorter in the $y$ direction, the growing dendrite tip reaches the outer boundary in that direction. As in the previous calculations, this one was performed on half the domain shown and was reflected about the vertical centerline. For the calculation, $X_{L}=9.0$ and $Y_{L}=4.5$ with 600 grid points in the $x$ direction and 300 points in the $y$ direction yielding $\Delta X=\Delta Y=0.015$. The time-step was $1 \times 10^{-4}$.

Finally, we present a set of calculations with six-fold anisotropy $(k=6)$ in fig. 5. Unlike the previous calculations, a square domain with $X_{L}=Y_{L}=9$ has been used. There are 900 grid points in each direction and the time-step was again $1 \times 10^{-4}$. The calculation was initiated by a small circular region of solid located in the center of the domain. The anisotropy level is $\gamma=0.01$, and a $1 \%$ level of random noise is used to facilitate the occurrence of irregular side branching. The development of the dendritic structure with six-fold primary structure is shown in the figure at the four dimensionless time values: $0.1,0.2,0.4$, and 0.6. The occurrence of secondary side-branches and even some tertiary branch formation is clearly displayed in the simulation as well as the inclusion of a liquid pocket. Also evident in this figure and in the previous one is the occurrence of dendrite side-arm coarsening. All these features and the fact that the calculations produce a well-defined operating state for the dendrite tip are good examples of how the phase-field model can produce realistic dendritic growth behavior.

The results presented here demonstrate the complexity of the solidification morphology that can be simulated using the phase-field approach. This work is part of an ongoing effort to evaluate the behavior of phase-field models both qualitatively and quantitatively, in order to access their utility as a practical tool for computing solidification growth morphologies of real materials under a variety of conditions.

\section{Acknowledgements}

This work was performed with partial support from the Applied and Computational Mathematics Program of the Defense Advanced Research Projects Agency. One of the authors (AAW), gratefully acknowledges partial support from a NATO collaborative research grant. 


\section{References}

[1] Langer, J. S., "Models of Pattern Formation in First-Order Phase Transitions", in Directions in Condensed Matter Physics, edited by G. Grinstein and G. Mazenko, (World Scientific, Philadelphia, 1986), pp. 165-186.

[2] Collins, J. B. and Levine, H., "Diffusion interface model of diffusion-limited growth", Phys Rev. B, Vol. 31, 1985, 6119-6122.

[3] Caginalp, G., "Surface tension and supercooling in solidification theory", in Applications of Field Theory to Statistical Mechanics, edited by L. Garrido, Lecture Notes in Physics No. 216 (Springer-Verlag, Berlin, 1985), pp. 216-226.

[4] Caginalp, G., "An Analysis of a Phase Field Model of a Free Boundary", Arch. Rat. Mech. Anal., Vol. 92, 1986, pp. 205-245.

[5] Caginalp, G., "Stefan and Hele-Shaw type models as Asymptotic Limits of the PhaseField Equations", Phys. Rev. A, Vol. 39, 1989, pp. 5887-5896.

[6] Cahn, J. W. and Hilliard, J. E., "Free energy of a nonuniform system. I. Interfacial free energy", J. Chem. Phys., Vol. 28, 1958, pp. 258-267.

[7] Cahn, J. W., "On spinodal decomposition", Acta Metall., Vol. 9, 1961, pp. 795-801.

[8] Allen, S. M. and Cahn, J. W., "A microscopic theory for antiphase boundary motion and its application to antiphase boundary domain coarsening", Acta Mettall., Vol. 27, 1979, pp. 1085-1095.

[9] Halperin, B. I., Hohenburg, P. C. and Ma, S.-K., "Renormalization group methods for critical dynamics: I. Recursion relations and the effects of energy conservation", Phys. Rev. B, Vol. 10, 1974, pp. 139-153.

[10] Fix, G. J., "Phase field methods for free boundary problems", in Free boundary problems: theory and applications Vol. II, edited by A. Fasano and M. Primicerio (Pitman, Boston, 1983), pp. 580-589.

[11] Lin, J.-T., "The Numerical Analysis of a Phase Field Model in Moving Boundary Problems", Doctoral Thesis, Dept. of Mathematics, Carnegie Mellon University, 1984. 
[12] Caginalp, G. and Socolovsky, E. A., "Computation of Sharp Phase Boundaries by Spreading: The Planar and Spherically Symmetric Cases", J. Comp. Phys., Vol. 95, 1991, pp. 85-100.

[13] Schofield, S. A. and Oxtoby, D. W., "Diffusion disallowed crystal growth. I. LandauGinzburg model", J. Chem. Phys., Vol. 94, 1991, pp. 2176-2186.

[14] Kobayashi, R., "Simulations of Three-Dimensional Dendrites", preprint and associated video tape, 1992.

[15] Kobayashi, R., "Modeling and Numerical Simulations of Dendritic Crystal Growth", to appear in Physica D, 1993.

[16] Wheeler, A. A., Murray, B. T. and Schaefer, R. J., "Computation of Dendrites Using a Phase-Field Model", to appear in Physica D, 1993.

[17] Wang, S.-L., Sekerka, R. F., Wheeler, A. A., Murray, B. T., Coriell, S. R., Braun, R. J. and McFadden, G. B., "Thermodynamically-Consistent Phase-Field Models for Solidification", submitted to Physica D.

[18] Penrose, O. and Fife, P. C., "Thermodynamically-Consistent Models of Phase-Field Type for the Kinetics of Phase Transitions", Physica D, Vol. 43, 1990, pp. 44-62.

[19] Wheeler, A. A., Boettinger, W. J. and McFadden, G. B., "Phase-Field model for isothermal phase transitions in binary alloys", Phys. Rev. A, Vol. 45, 1992, pp. 7424-7439.

[20] Wheeler, A. A., Boettinger, W. J. and McFadden, G. B., "A phase-field model of Solute Trapping during solidification", to appear in Phys. Rev. A, 1993.

[21] McFadden, G. B., Wheeler, A. A., Braun, R. J., Coriell, S. R., and Sekerka, R. F, "Phase-Field Models for Anisotropic Interfaces", submitted to Phys. Rev. A.

[22] Roache, P. J., Computational Fluid Dynamics (Hermosa, Albuquerque, N. M., 1976).

[23] Saito, Y., Goldbeck-Wood, G. and Mueller-Krumbhaar, H., "Numerical Simulation of Dendritic Growth", Phys. Rev. A, Vol. 38, 1993, pp. 2148-2157. 


\section{Figure Captions}

Figure 1. The computed isotherms (left) and phase-field contours (right) at three time levels: $0.1,0.3$, and 0.5 (shown from bottom to top). The calculation is for no anisotropy, $\gamma$ $=0$. Note that the computations are symmetric about the vertical centerline.

Figure 2. The computed isotherms (left) and phase-field contours (right) at three time levels: $0.1,0.3$, and 0.5 (shown from bottom to top). The calculation has four-fold anisotropy $(k=4)$ with $\gamma=0.02$. Note that the computations are symmetric about the vertical centerline.

Figure 3. The computed isotherms (left) and phase-field contours (right) at a single time level 0.6 for a calculation with four-fold anisotropy $(k=4)$ and $\gamma=0.02$. Note that the computations are symmetric about the vertical centerline.

Figure 4. The computed isotherms (left) and phase-field contours (right) at four time levels: $0.1,0.3,0.9$ and 1.5 (shown from bottom to top). The calculation has four-fold anisotropy $(k=4)$ with $\gamma=0.01$ and a $1 \%$ random noise level. The computations are symmetric about the vertical centerline.

Figure 5. The computed isotherms (left) and phase-field contours (right) at four time levels: $0.1,0.2,0.4$ and 0.6 (shown from bottom to top). The calculation has six-fold anisotropy ( $k$ $=6)$ with $\gamma=0.01$ and a $1 \%$ random noise level. 

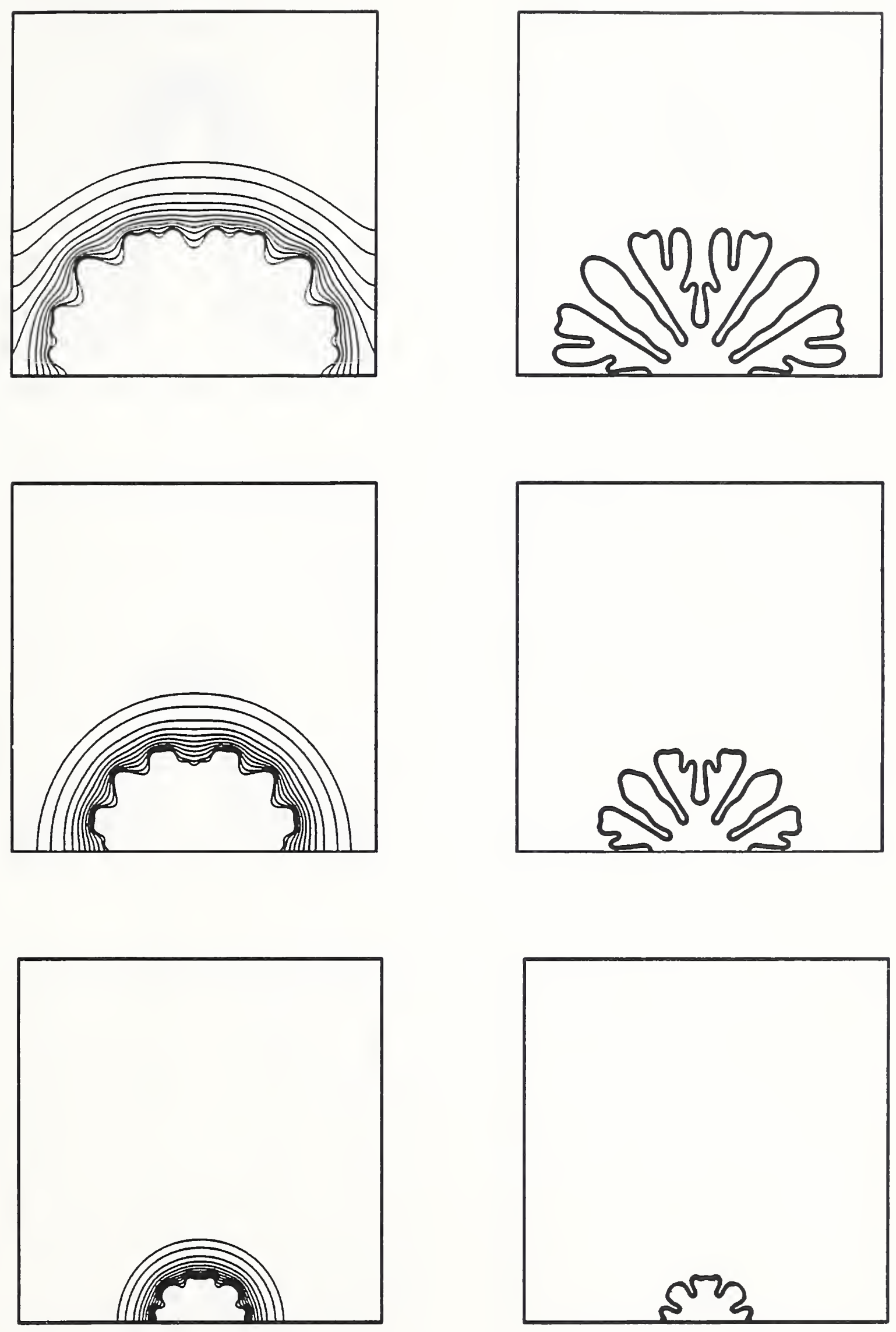

Fig. 1 


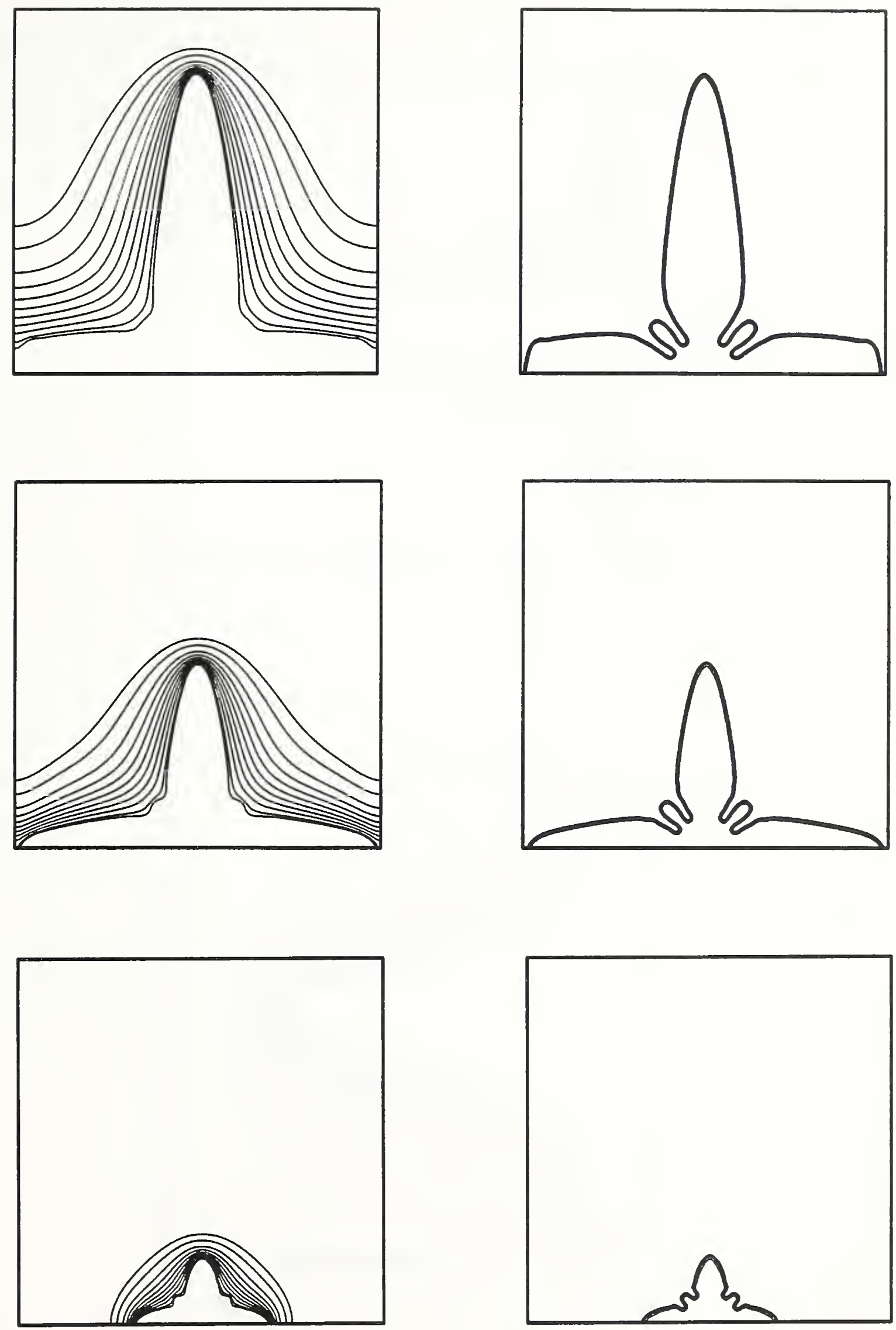

Fig. 2 


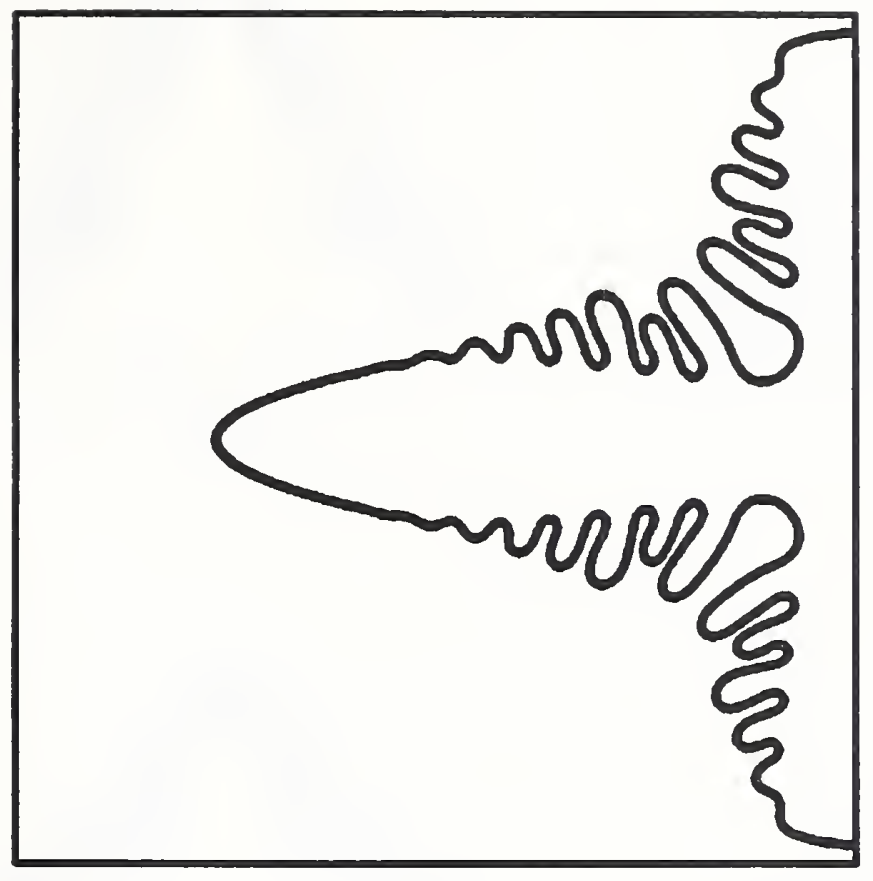

$m$
0
0
in

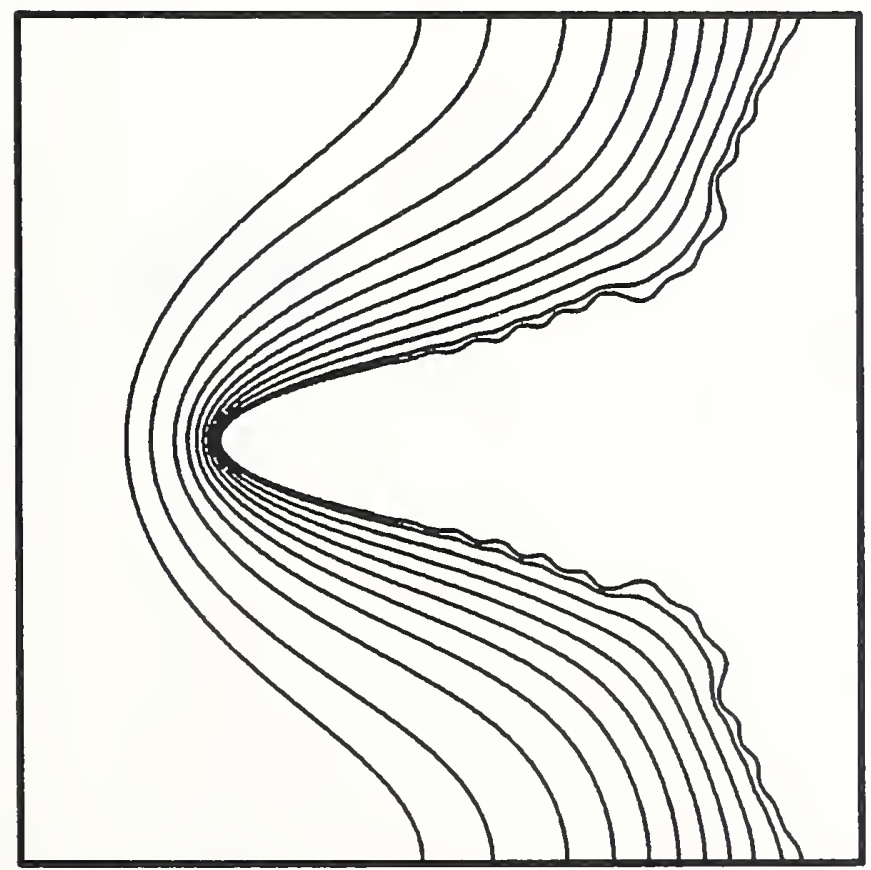



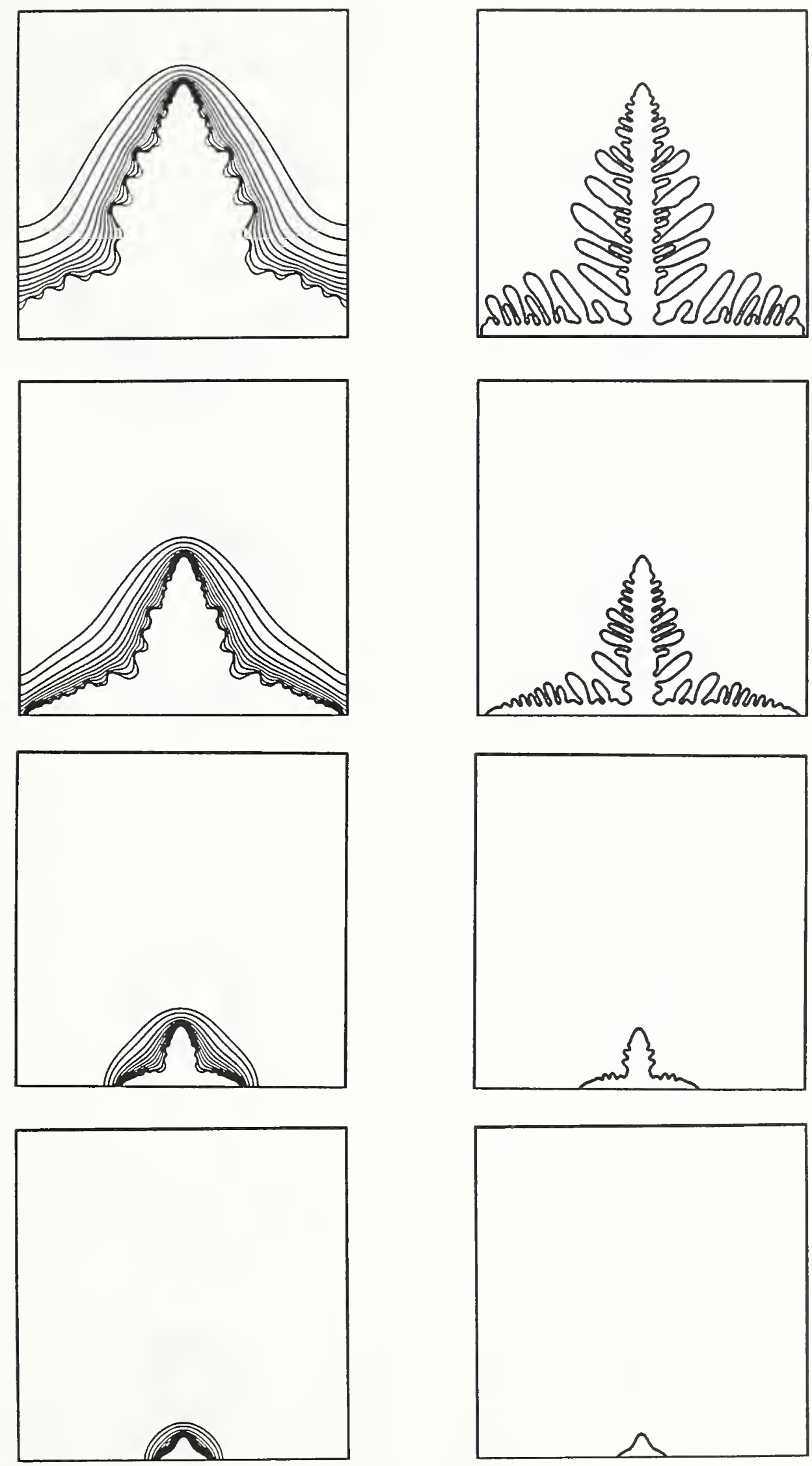

Fig. 4 

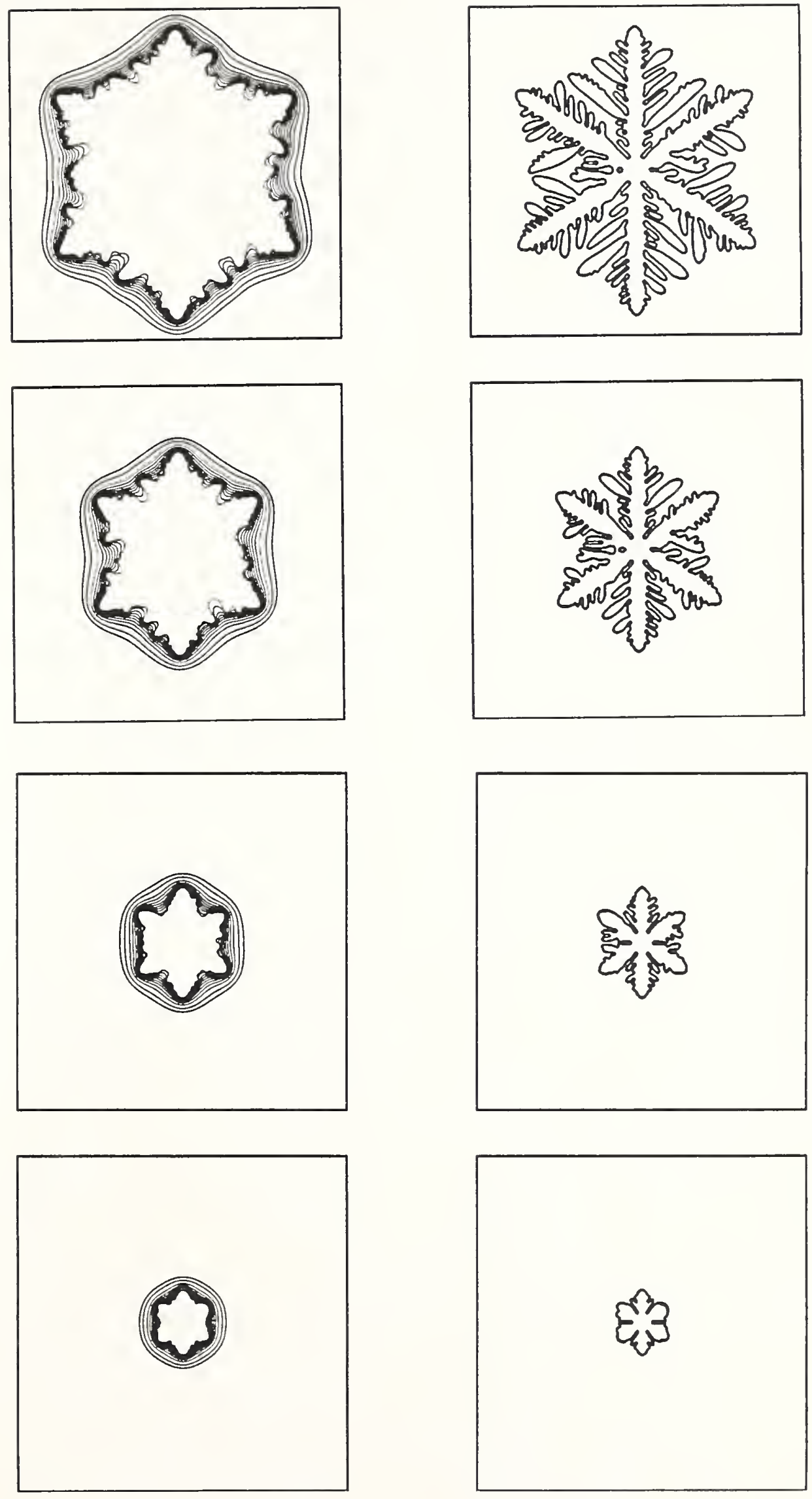

Fig. 5 

